Mortazavi, F., Mehrabadi, M., Hollins Martin, C.J., Martin, C.R. (2020) Psychometric Properties of the Birth Satisfaction Scale-Revised (BSS-R) in a Sample of Postpartum Iranian Women. Health Care for Women International.

https://doi.org/10.1080/07399332.2020.1802464

Psychometric Properties of the Birth Satisfaction Scale-Revised (BSS-R) in a Sample of Postpartum Iranian Women

Forough Mortazavi, Associate Professor in Reproductive Health, Department of Midwifery, School of Nursing \& Midwifery, Sabzevar University of Medical Sciences, Towhidshahr Avenue, Pardis Building, Sabzevar, Iran Email: frmortazavi@yahoo.com

Maryam Mehrabadi, MSc of Midwifery, Health Chancellery, Sabzevar University of Medical Sciences, Tabas square, Sabzevar, Iran $\quad$ Email: maryammehrabady@gmail.com

Caroline J. Hollins Martin, Professor in Maternal Health, School of Health and Social Care, Edinburgh Napier University (ENU), Sighthill Campus, Edinburgh, Scotland, UK, EH11 4BN, Email: c.hollinsmartin@napier.ac.uk

Colin R. Martin, Institute for Clinical and Applied Health Research (ICAHR) Rm 329, Allam Medical Building, University of Hull, Hull, HU6 7RX, UK Email: c.r.martin@hull.ac.uk

Corresponding author:

Forough Mortazavi, Associate Professor in Reproductive Health, Department of Midwifery, School of Nursing \& Midwifery, Sabzevar University of Medical Sciences, Towhidshahr Avenue, Pardis Building, Sabzevar, Iran Email: frmortazavi@yahoo.com 


\title{
Psychometric Properties of the Birth Satisfaction Scale-Revised (BSS-R) in a Sample of Postpartum Iranian Women
}

\begin{abstract}
Childbirth satisfaction may positively affect maternal intention to have a future pregnancy and preference to have a cesarean. We translate the UK-Birth-Satisfaction-Scale-Revised (UK-BSS-R) into Persian and validate an Iranian-BSS-R for future use in this population. In total, 784 mothers who were hospitalized in postpartum wards completed the Persian version of the BSS-R. The confirmatory factor analysis on 10-items scale confirmed the conventional three-factor structure. The Cronbach's alpha of the Iranian-BSS-R subscales and the total score were within the range of $0.53-0.76$. Our findings provide further evidence that the BSS-R is an internationally reliable and valid instrument to measure birth satisfaction.
\end{abstract}

Keywords: natural childbirth, patient satisfaction, postpartum, validation studies, psychometric, factor analysis 


\section{Psychometric Properties of the Birth Satisfaction Scale-Revised (BSS-R) in a Sample of Postpartum Iranian Women}

The declining trend of population growth in Iran began in 1990 with the implementation of the population control policy and family planning programs. The total fertility rate (TFR) in Iran was reported to be $6.5 \%$ and $1.8 \%$ in 1990 and 2005, respectively, which remained steady until 2012 (Cincotta \& Sadjadpour, 2017; Karamouzian, Sharifi, \& Haghdoost, 2014), prompting policymakers to shift their focus on women's fertility and TFR. During 1976-2010, the overall incidence of cesarean section increased from $19.5 \%$ to $48 \%$ in Iran, which in turn augmented the level of health problems in women and led to fertility reduction (Setudezadeh \& Yousefinezhadi, 2019). Therefore, health policies changed in favor of decreasing the rate of cesarean section and encouraging women to have more children. Consequently, studies in this regard have been mainly focused on the causes of low maternal fertility and high cesarean section rates (Setudezadeh \& Yousefinezhadi, 2019). Accordingly, factors such as the fear of childbirth, maternal negative attitude toward natural childbirth, and inappropriate childbirth physical environment are among the primary causes of maternal request for cesarean section (Darsareh, Aghamolaei, Rajaei, \& Madani, 2017).

In 2014, a major health policy known as the health transformation plan (HTP) was launched in the Iranian health system to promote public health. One of the HTP packages was set to reduce the rate of cesarean section in both private and public hospitals (Moradi-Lakeh \& VosooghMoghaddam, 2015). Therefore, numerous interventions were implemented in this regard to improve the infrastructures of maternity facilities and childbirth equipment in hospitals, provide proper delivery facilities in the format of labor, delivery, recovery postpartum model (LDRP), hold free childbirth preparatory classes in hospitals, and provide free hospital birth services. In addition, financial incentives for normal delivery providers in public hospitals have been 
proposed (Moradi-Lakeh \& Vosoogh-Moghaddam, 2015; Setudezadeh \& Yousefinezhadi, 2019), so that after 15 months, the Ministry of Health announced that the rate of cesarean section reduced to 10\% (Rashidian et al., 2019; Shahshahan, Heshmati, Akbari, \& Sabet, 2016). However, a significant reduction could not be predicted in the rate of cesarean section in the long run (Rashidian et al., 2019). Along with these measures, the effects of some interventions (e.g., preparatory classes) on the tendency toward natural delivery (khavandizadeh Aghdam, Mahfouzi, \& Kazemzadeh, 2019) and fear of childbirth in women (Karrabi, Farjamfar, Mortazavi, Nazari, \& Goli, 2019) have been investigated, while there have been no studies on the outcomes of HTP (e.g., maternal birth satisfaction).

Childbirth is one of the most significant medical events in a woman's life. One of the main roles of childbirth care providers is to help women to have a pleasant birth experience. A recent study in Chili indicates that patient-provider interaction during labor and the physical environment are the most important predictors of maternal birth satisfaction (Pantoja et al., 2020). According to the literature, childbirth dissatisfaction with normal delivery or cesarean section reduces the likelihood of subsequent pregnancies (Rashidian et al., 2019). Another negative consequence of dissatisfaction with natural childbirth is increased maternal tendency toward cesarean section and increased likelihood of both emergency and elective cesarean section (Johnson \& Slade, 2002).

As patient satisfaction is considered to be an important indicator of healthcare quality assessment in the world (Mitchell, Ferketich, \& Jennings, 1998), the Iranian Ministry of Health has fulfilled one of its most important missions since 2011 by obliging hospitals to review patient satisfaction with hospital services and the received care, while implementing proper measures to increase patient satisfaction (Yousefinezhadi, Mosadeghrad, Arab, Ramezani, \& 
Sari, 2017). Therefore, women's satisfaction with childbirth has received considerable attention in Iran.

Measurement of women's satisfaction with childbirth requires valid, reliable, and multidimensional instruments. In this regard, the 30-item Birth Satisfaction Scale (BSS) was designed (Hollins Martin \& Fleming, 2011). In their study, three themes emerged, including the quality of the provided care, individual characteristics, and experienced stress during labor. In 2014, the 10-item version of the Birth Satisfaction Scale-Revised (BSS-R) was developed from the 30-item version. According to the findings, the short version had acceptable validity and reliability and is now recommended by the International Consortium for Health Outcome Measures (ICHOM) as the international measure of choice for assessing women's experiences of childbirth (Martin \& Martin, 2014).

Various tools have been developed to assess childbirth satisfaction, while only few have been translated into Persian. In this regard, a study in Tehran (Iran) entitled the "Psychometric Assessment of the Ganger 40-item Normal Delivery and cesarean Satisfaction Tools was conducted in 2016" (Pakari, Tork Zahrani, Nasiri, \& Mahmoodi, 2016). Furthermore, the Mackey and Goodman 34-item delivery satisfaction scale was translated and validated in 2016 (Moudi \& Tavousi, 2016). The psychometric properties of the six-item Persian BSS-R indicator were confirmed in Tehran, while the 10-item BSS-R has been introduced as an internationally recommended tool by the ICHOM, allowing comparison of maternal satisfaction across countries. One of the key advantages of this tool is the lowest number of the items and good validity indices.

Considering that birth experience is linked to the intentions of women for future pregnancy, use of validated birth satisfaction scales in Iran is the first step towards recognition of influential factors in childbirth satisfaction and adopting proper strategies to optimize women's experiences. In addition, such tools could help compare maternal satisfaction with natural 
delivery and cesarean section on a national scale. In Iran, the importance of these tools is further emphasized due to the high rate of cesarean section (Gibbons et al., 2010) and increased maternal request for cesarean section without medical indications (Darsareh et al., 2017). As such, the reduction of cesarean section is a priority of the Iranian Ministry of Health.

The aims of the researchers were to translate the 10-item UK-BSS-R and determine its validity and reliability in a population of Iranian postpartum women. Lack of a validated and short scale for the assessment of birth satisfaction has made it impossible to assess the effects of the measures taken nationally, which may lead to the disregard of maternal birth satisfaction and its incorporation into the policies regarding cesarean rate reduction and fertility rate growth in Iran.

\section{Materials and Methods}

The researchers conducted this study in two phases. In the first phase, we translated the instrument, and examined its content validity using the MAPI research institute linguistic validation guidelines. In the second phase, we performed a descriptive, cross-sectional study involving 816 mothers who had experienced childbirth within the past 24 hours prior to the day of the interview. The sample population included new mothers who were hospitalized in the postpartum and postoperative wards of Mobini Hospital, which is the only maternity hospital in Sabzevar city (Iran) and is affiliated to Sabzevar University of Medical Sciences. Mobini Hospital is an infant-and mother-friendly hospital with about 500 births per month. However, the delivery ward is not in the LDRP. We performed a convenience sampling at this stage.

The inclusion criteria of the study were as follows: 1) uncomplicated pregnancy with healthy, single infants; 2) childbirth within the past 24 hours prior the interview; 3) basic literacy; 4) physical preparedness to complete the questionnaires and 5) consent to participate in the study. The exclusion criteria were as follows: 1) fetal death or congenital anomalies in the current pregnancy; 2) history of chronic diseases; 3) of mental health disorders; 4) presence of 
antepartum/postpartum complications (e.g., severe postpartum hemorrhage, severe fever, hypertension/preeclampsia) or other conditions adversely affecting maternal psychological health or reducing the likelihood of completing the questionnaire accurately.

The study was performed between June-September 2019. The first author worked with two newly graduated midwives to recruit postnatal mothers and collect data. After obtaining verbal consent to participate in the study, participants were asked to complete the sociodemographic questionnaire (i.e., age, occupation status, education level, income status, and parity), BSS-R, and Wijma Delivery-Expectancy Questionnaire (W-DEQ) version B.

Post providing written and informed consent, documentation was retained in the participants file for future availability to the midwife who asked the questions. Telephone number and address of the first author was included in the consent form. To determine sample size, we collected data from 296 participants and divided them into two groups: (1) cesarean section $(n=82)$ and (2) natural delivery $(n=214)$, and considered the effect size of 0.23 for further analysis (Martin \& Martin, 2014). To achieve the test power of 0.80 and alpha of 0.05 , we determined the sample size to be 210 for the cesarean group, 546 for the natural delivery group, and 756 for the total sample. Considering 5\% incomplete questionnaire rate and $2 \%$ outliers, we calculated the final sample size to be 810 . The Ethics Committee of Sabzevar University of Medical Sciences approved the study protocol (ethics code: IR. MEDSAB.REC.1397.119).

\section{Content Validity}

In the first phase of the study, one doctoral graduate in reproductive health and one doctoral graduate in English language translated the BSS-R into Persian. We compared the two translated versions, and made some revisions to the first version of the BSS-R. For instance, one of translators translated 'labor and delivery' to 'delivery stages', and the other had translated the same phrase to 'labor stage and giving birth stage'; therefore, we agreed on the phrase 'delivery stages'. Following that, two doctoral graduates in English language translated the first 
version of the Iranian-BSS-R into English. Finally, the researchers assessed the two translations and prepared the second Persian version of the questionnaire.

In the next phase of the research, 10 experts in the fields of reproductive health, midwifery, and health promotion, who were familiar with the research and scale adoption, evaluated the contents of the Iranian-BSS-R. In the qualitative phase, experts reviewed each item of the scale, and their general views were obtained on both appearance of the scale and relevance of the items to Iranian culture. Furthermore, one expert commented that the third item was vague, since Iranian women in labor do not have explicit choices regarding their delivery process. However, others argued that the documented choices of women are to be encompassed soon. Midwives often explain choices available to childbearing women verbally. The experts recommended that we change the presentation of the items options, so that the option 'Strongly Disagree' would become 'Not at All'. In addition, they recommended that only the options 'Not at All' and 'Strongly Agree' be presented at the first row of the Iranian-BSS-R, and the other options change to one, two, and three values. In addition, the experts suggested that item nine (I was not distressed at all during labor.), which was the only item with negation, be modified to an item with a positive verb in agreement with the other items; others also agreed with these modifications

In the quantitative phase, the experts evaluated the necessity of each item by selecting an option from among three options of unnecessary, useful but unnecessary, and necessary. We calculated the content validity ratio (CVR), and the results indicated that the experts considered all the items to be necessary (CVR $>0.62$ for each item) (Lawshe, 1975). In addition, the experts evaluated the scale in terms of its simplicity and transparency of items based on a four-point scale (Not Simple and Not Clear: 1, Very Simple and Very Clear: 4). We also calculated the content validity index (CVI) for each item. No item had lower CVI than 0.8 for simplicity and transparency (Polit \& Beck, 2004). 
As for face validity, the first author asked 10 postpartum women with low education levels to complete the instrument. Afterwards, the first author interviewed these women, and they claimed no specific difficulty in completing the questionnaire. On average, participants completed the scale within three minutes, and no comments were received on the items. A Persian language editor revised the final version of the questionnaire.

\section{Instruments}

\section{The Birth Satisfaction Scale-Revised (BSS-R)}

BSS-R was developed to measure birth satisfaction in the postpartum period (Martin \& Martin, 2014). This scale consists of 10-items that participants respond to on a five-point Likert scale which ranges from 0-4 (Strongly Disagree to Strongly Agree). The minimum and maximum scores of the questionnaire are zero and 40, respectively. The reliability of the 10-item scale has been determined to be 0.79 . In the present study, CFA indicated that the scale contains three factors, which include stress experienced during labor (four items), women's personal attributes (two items), and quality of care provision (four items). The divergent validity of the BSS-R was confirmed by the non-significant correlation of the BSS-R total score and maternal age $(\mathrm{r}=0.07$; $\mathrm{P}=0.27$ ). The scale has been translated and validated in different languages, including Italian (Nespoli, Colciago, Pedroni, Perego, \& Fumagalli, 2018), Turkish, (Goncu Serhatlioglu, Karahan, Hollins Martin, \& Martin, 2018), Slovak (Skodova, Nepelova, Grendar, \& Baskova, 2019), and Spanish (Romero-Gonzalez et al., 2019). Furthermore, the validity of the scale has been confirmed in the Australian culture (Jefford, Hollins Martin, \& Martin, 2018) and US context (Barbosa-Leiker, Fleming, Hollins Martin, \& Martin, 2015).

\section{Wijma Delivery Expectancy Questionnaire (W-DEQ Version B)}

W-DEQ version B has been developed to investigate fear of childbirth during the postpartum period (Wijma, Wijma, \& Zar, 1998). The W-DEQ contains 33 items, which are scored based on a six-point scale that ranges between 0-5 (Not at All to Extremely). The minimum and 
maximum total scores of the questionnaire are zero and 165, respectively, with higher scores indicating higher levels of childbirth fear. Internal consistency of the scale using Cronbach's alpha $=0.93$, and validity has been confirmed by moderate correlations of the scale with several other psychological inventories. In addition, the two cutoff points of 85 and 100 have been proposed for the screening of women with moderate-to-severe childbirth fear, respectively. The scale has been translated into Persian, and its validity and reliability have been confirmed. The scale has also demonstrated proper reliability and acceptable construct validity (Mortazavi, 2017).

\section{Statistical Analysis}

Data analysis was performed in R version 3.6.1, LISREL version 8.5, and SPSS version 18 . We evaluated the normality of the data. We used descriptive tests (means, ranges, skewness, and kurtosis) to define the sample characteristics and distribution of the women's responses to the questionnaire items.

\section{Confirmatory Factor Analysis (CFA)}

In the current study, we used CFA (Brown, 2015) to examine the tridimensional measurement model proposed by the scale developers (Martin \& Martin, 2014). We used Maximumlikelihood estimation to evaluate the CFA model (Brown, 2015), and evaluated two CFA models, including one unidimensional model and one tridimensional model. We used the multiple goodness-of-fit tests to evaluate the models (Bentler \& Bonett, 1980). We considered the root mean square error of approximation (RMSEA) of $<0.08$, CFI of $0.90 \leq$ (Bentler, 1990), and standardized root mean square residual (SRMR) of $<0.08$ (Hu \& Bentler, 1999) as the acceptable model fit.

We examined the correlation-coefficients of the Iranian-BSS-R subscales and total score and compared with the original $U K-B S S-R$ validation study as another evidence for the construct validity. 


\section{Internal Consistency}

We used the Cronbach's alpha coefficient to assess the reliability of the scale, and the values of $>0.7$ were considered acceptable (Streiner, 2003).

\section{Known-groups Discriminant Validity}

We evaluated Known-groups discriminant validity through comparing the Iranian-BSS-R subscales and total scores between elective cesarean section and emergency cesarean section groups. Moreover, we predicted the significantly higher total score and subscales of the Iranian$B S S-R$ in elective cesarean section group compared to the emergency cesarean section group.

\section{Divergent Validity}

To determine divergent validity of the Iranian-BSS-R, we calculated correlations between maternal age and scale total scores and its subscales, and we predicted no significant correlation between the Iranian-BSS-R scores and maternal age.

\section{Concurrent Validity}

We evaluated concurrent validity by examining the correlation-coefficients between the total score and subscale scores of the Iranian-BSS-R and W-DEQ version B total scores. We predicted statistically significant correlation-coefficients between the Iranian-BSS-R total and subscale scores with W-DEQ version B total scores.

\section{Results}

\section{Participants}

Among the 810 postnatal women who participated in the study, 800 cases completed the Iranian-BSS-R (response rate: 98\%). After removing the outliers, we determined the sample size to be 784 . The mean age, gestational age, and education level of the participants were $28.43 \pm 6.07$ years, $39.01 \pm 1.32$ weeks, and $11.22 \pm 3.65$ years, respectively. In total, $40.7 \%$ of the women were primiparous and $89.8 \%$ were housewives. The mode of delivery in $68.5 \%$ of the women was natural vaginal birth (table 1). 
Table 2 shows the mean and distributional characteristics of the individual Iranian-BSS-R items. The distribution of items on the Iranian-BSS-R demonstrated no significant evidence of skewness or kurtosis. According to findings, item 4 (I was distressed during labor.) and item 9 (Ifelt very anxious during my labor and childbirth.) had the lowest mean scores.

Table 3 shows the mean and distributional characteristics of the Iranian-BSS-R subscales and total score. The mean Iranian-BSS-R total score was $22.94 \pm 7.04$ out of 40 , and the highest mean score was observed in the subscale of maternal satisfaction with the quality of care.

\section{Internal Consistency}

The Cronbach's alpha of the Iranian-BSS-R subscales and total score was within the range of 0.53-0.76. We compared the Cronbach's alpha of the Iranian-BSS-R subscales and total score with the original $U K-B S S-R$ validation study (Martin \& Martin, 2014), and the Cronbach's alpha coefficients of the Iranian-BSS-R was not significantly lower than the original $U K-B S S$ $R$ validation study (Table 3 ).

\section{Confirmatory Factor Analysis (CFA)}

Table 4 and Figure 1 show the results of the CFA on the 10-item Iranian-BSS-R. Accordingly, the three-factor measurement model of the $U K-B S S-R$ was a good fit to the data. Figures 1 support the three-factor model. Standardized item-factor loadings are in the range of 0.17-0.92. All standardized item-factor loading are greater than 0.3 except one. T-values are in the range of 4.52-28.44. No T-value is $<2$, indicating that the relationships between each latent variable and its observed variables are statistically significant.

According to findings, correlations of the Iranian-BSS-R subscales and total score were not significantly different with to those of the original $U K-B S S-R$, with exception of the correlation between the women's attributes and Iranian-BSS-R total scores, as well as the correlation between women's attributes and quality of care (Table 5).

\section{Known-groups Discriminant Validity}


Table 6 shows comparison of the Iranian-BSS-R total and subscale scores differentiated by cesarean section type. Consistent with our prediction, the total and subscale scores were significantly higher in the elective cesarean section group compared to the emergency cesarean group.

\section{Divergent Validity}

We confirmed the divergent validity of the Iranian-BSS-R scale by the correlation between maternal age and Iranian-BSS-R total scores $(\mathrm{r}=0.05 ; \mathrm{P}=0.15)$. The Pearson's correlationcoefficient of maternal age and Iranian-BSS-R subscale scores of stress experienced $(\mathrm{r}=0.07$; $\mathrm{P}=0.06)$, women's attributes $(\mathrm{r}=0.02 ; \mathrm{P}=0.47)$, and quality of care $(\mathrm{r}=0.01 ; \mathrm{P}=0.72)$ were not considered significant.

\section{Concurrent Validity}

We confirmed concurrent validity by the significant, negative, moderate correlations between the Iranian-BSS-R subscales (stress experienced, women's attributes, and quality of care) and total scores with the W-DEQ version $\mathrm{B}$ total scores $(-0.40--0.68 ; \mathrm{P}<0.001)$. In addition, the stress experienced subscale had the most significant correlation with the W-DEQ version B.

\section{Discussion}

We aimed to translate and validate the $U K-B S S-R$ for measuring birth satisfaction in Iranian mothers. We translated the scale into Persian using the forward-backward method and a group of experts content-validated it. The satisfactory CVR and CVI of all the items indicated that the Iranian-BSS-R is proper for use in the Iranian context. Furthermore, our findings confirmed validity and reliability of the Iranian-BSS-R as an instrument for measuring birth satisfaction in Iranian women, providing further support for the robustness of the scale in terms of factor structure and reliability.

According to the results of the present study, internal consistency of the Iranian-BSS-R total score and subscales of quality of care and stress experienced was satisfactory and close to the 
threshold, respectively. In addition, the Cronbach's alpha coefficient of the women's attributed subscale (only two items) was not significantly lower than the original $U K-B S S-R$ validation study (Martin \& Martin, 2014).

In terms of validity, CFA indicated that the conventional three-factor model proposed by the scale developers (Martin \& Martin, 2014) was a good fit to the data. On the other hand, our findings demonstrated that the model fit for the unidimensional Iranian-BSS-R was poor. These findings are in line with previous validation studies conducted in the UK (Martin \& Martin, 2014), United States (Barbosa-Leiker et al., 2015), Australia (Jefford et al., 2018), Spain (Romero-Gonzalez et al., 2019), and Turkey (Goncu Serhatlioglu et al., 2018).

According to the findings of the current research, the Iranian-BSS-R total and subscale scores were higher in the elective cesarean section group compared with the emergency cesarean section group. These findings are also consistent with results of a further US study involving 160 women who had natural vaginal birth and 44 cesarean sections. In the postpartum period, women planning cesarean section often report their highest satisfaction and lowest scores for distress. In addition, no significant difference has been denoted between planned natural vaginal birth and planned cesarean section (Blomquist, Quiroz, Macmillan, McCullough, \& Handa, 2011).

The results of the present study demonstrated no significant correlations between maternal age and the Iranian-BSS-R total and subscale scores, which confirmed the divergent validity of the scale. In all the previous validation studies on the BSS-R, divergent validity has been confirmed (Barbosa-Leiker et al., 2015; Goncu Serhatlioglu et al., 2018; Martin \& Martin, 2014; RomeroGonzalez et al., 2019).

This study is the first step towards helping maternity care professionals in Iran assess birth satisfaction, with further research recommended to reveal weaknesses in maternity care and 
investigate correlates and predictors of birth satisfaction. Furthermore, the Iranian-BSS-R could be used to evaluate birth satisfaction in the postpartum period in a variety of settings.

The main strengths of the present study are its large sample size (both primiparous and multiparous women) and hospital-based recruitment. In addition, we assessed birth satisfaction within the first 24 hours of delivery, which may prevent recalling bias.

\section{Limitations}

The limitations of this study include usual limitations involved in the use of self-administered scales. Participants' responses may be influenced by what they consider to be socially acceptable norms. In addition, they may not be able to assess their own birth satisfaction accurately.

\section{Conclusion}

In the current research, we translated the $U K-B S S-R$ from English into Persian and investigated its validity and reliability. The Iranian-BSS-R is now a short, easy to use, practical, reliable, and valid scale for measuring birth satisfaction in Iranian postpartum women. The scale can now be used as part of the routine assessment of birth satisfaction in the postpartum period. This newly validated Iranian-BSS-R provides opportunity for Iranian researchers, maternal healthcare professionals and policymakers to compare maternal satisfaction between natural childbirth and cesarean section. In addition, the Iranian-BSS-R can be used to evaluate interventions designed to improve childbearing women's experiences of childbirth. To conclude, this study provides further evidence that the BSS-R is an internationally reliable and valid instrument for measuring birth satisfaction.

\section{Acknowledgments}

We thank all the mothers who participated in our study.

\section{Declaration of interest statement}

The authors report no conflict of interest.

\section{Resources}


The Research Vice-Chancellery of Sabzevar University of Medical Sciences approved the study proposal. This work was supported by the financial assistance from Sabzevar University of Medical Sciences Research Council under Grant (97249). 


\section{References}

Barbosa-Leiker, Celestina, Fleming, Susan, Hollins Martin, Caroline J., \& Martin, Colin R. (2015). Psychometric properties of the Birth Satisfaction Scale-Revised (BSS-R) for US mothers. Journal of Reproductive and Infant Psychology, 33(5), 504-511. doi: 10.1080/02646838.2015.1024211

Bentler, P. M. (1990). Comparative fit indexes in structural models. Psychol Bull, 107(2), 238-246. PMID: 2320703

Bentler, P. M., \& Bonett, Douglas G. (1980). Significance tests and goodness of fit in the analysis of covariance structures. Psychol Bull, 88(3), 588-606. . doi: 10.1037/00332909.88.3.588

Blomquist, J. L., Quiroz, L. H., Macmillan, D., McCullough, A., \& Handa, V. L. (2011). Mothers' satisfaction with planned vaginal and planned cesarean birth. Am J Perinatol, 28(5), 383-388. doi: 10.1055/s-0031-1274508

Brown, T. (2015). Confirmatory Factor Analysis for Applied Research (2nd ed.). New York Guilford Press.

Cincotta, Richard , \& Sadjadpour, Karim (2017). IRAN IN TRANSITION: The Implications of the Islamic Republic's Changing Demographics. Washington, DC: Carnegie Endowment for International Peace.

Darsareh, Fatemeh, Aghamolaei, Teamur, Rajaei, Minoo , \& Madani, Abdoulhossain (2017). Determinants of caesarean birth on maternal demand in the Islamic Republic of Iran: a review. Eastern Mediterranean Health Journal, 23(6), 441-448.

Gibbons, Luz, Belizán, José M., Lauer, Jeremy AAna, Betrán, P , Merialdi, Mario, \& Althabe, Fernando (2010). The Global Numbers and Costs of Additionally Needed and Unnecessary Caesarean Sections Performed per Year: Overuse as a Barrier to Universal Coverage Geneva: WHO

Goncu Serhatlioglu, S., Karahan, N., Hollins Martin, C. J., \& Martin, C. R. (2018). Construct and content validity of the Turkish Birth Satisfaction Scale - Revised (T-BSS-R). $J$ Reprod Infant Psychol, 36(3), 235-245. doi: 10.1080/02646838.2018.1443322

Hollins Martin, Caroline, \& Fleming, Valerie. (2011). The birth satisfaction scale. International Journal of Health Care Quality Assurance, 24(2), 124-135. doi: doi:10.1108/09526861111105086

Hu, Li-tze, \& Bentler, Peter M. (1999). Cutoff criteria for fit indexes in covariance structure analysis: Conventional criteria versus new alternatives. Struct Equ Modeling, 6(1), 155. . doi: 10.1080/10705519909540118

Jefford, E., Hollins Martin, C. J., \& Martin, C. R. (2018). Development and validation of the Australian version of the Birth Satisfaction Scale-Revised (BSS-R). J Reprod Infant Psychol, 36(1), 42-58. doi: 10.1080/02646838.2017.1396302

Johnson, R., \& Slade, P. (2002). Does fear of childbirth during pregnancy predict emergency caesarean section? BJOG, 109. doi: 10.1046/j.1471-0528.2002.01351.x

Karamouzian, Mohammad, Sharifi, Hamid , \& Haghdoost, Ali Akbar. (2014). Iran's shift in family planning policies: concerns and challenges. Int J Health Policy Manag 3(5), 231-233.

Karrabi, Razieh, Farjamfar, Maryam, Mortazavi, Foroughossadat, Nazari, Ali Mohammad, \& Goli, Shahrbanoo. (2019). The effect of solution-focused group counseling on pregnant women's worries: A randomized clinical trial. Hayat, 25(1), 81-94.

khavandizadeh Aghdam, Sima, Mahfouzi, Yalda, \& Kazemzadeh, Rafat. (2019). Effect of prenatal preparation classes on the duration of labor and delivery type in primiparous women. The Iranian Journal of Obstetrics, Gynecology and Infertility, 21(11), 37-43. doi: 10.22038/ijogi.2019.12324 
Lawshe, CH. (1975). A quantitative approach to content validity. Pers Psychol, 28, 563-575. doi: 10.1111/j.1744-6570.1975.tb01393.x

Martin, Caroline J. Hollins, \& Martin, Colin R. (2014). Development and psychometric properties of the Birth Satisfaction Scale-Revised (BSS-R) Midwifery, 30, 610-619. doi: http://dx.doi.org/10.1016/j.midw.2013.10.006

Mitchell, P. H., Ferketich, S., \& Jennings, B. M. (1998). Quality health outcomes model. American Academy of Nursing Expert Panel on Quality Health Care. Image J Nurs Sch, 30(1), 43-46.

Moradi-Lakeh, M, \& Vosoogh-Moghaddam, A. (2015). Health sector evolution plan in Iran; equity and sustainability concerns. Int J Health Policy Manag., 4(10), 637-640.

Mortazavi, F. (2017). Validity and reliability of the Farsi version of Wijma delivery expectancy questionnaire: an exploratory and confirmatory factor analysis. Electronic Physician, 9(6), 4616-4615. doi: http://dx.doi.org/10.19082/4606

Moudi, Z., \& Tavousi, M. (2016). Evaluation of Mackey Childbirth Satisfaction Rating Scale in Iran: What Are the Psychometric Properties? Nurs Midwifery Stud, 5(2), e29952. doi: $10.17795 /$ nmsjournal29952

Nespoli, A., Colciago, E., Pedroni, S., Perego, S., \& Fumagalli, S. (2018). The Birth Satisfaction Scale-Revised (BSS-R): process of translation and adaptation in an Italian context. Ann Ist Super Sanita, 54(4), 340-347. doi: 10.4415/ann_18_04_11

Pakari, Nadieh, Tork Zahrani, Shahnaz, Nasiri, Maliheh , \& Mahmoodi, Zohrerh (2016). Persian Translation and Psychometric Testing of the Scales for Measuring Maternal Satisfaction in Normal and Caesarean Birth. Biosci Biotech Res Asia 13(1). doi: http://dx.doi.org/10.13005/bbra/2038

Pantoja, Loreto, Weeks, Fiona H., Ortiz, Jovita, Cavada, Gabriel, Foster, Jennifer, \& Binfa, Lorena. (2020). Dimensions of childbirth care associated with maternal satisfaction among low-risk Chilean women. Health Care for Women International, 41(1), 89-100. doi: 10.1080/07399332.2019.1590360

Polit, DF, \& Beck, CT (2004). Nursing Research: Principles and Methods (46th ed.). Philadelphia, USA: Lippincott

Rashidian, A., Moradi, G., Takian, A., Sakha, M. A., Salavati, S., Faraji, O., \& Piroozi, B. (2019). Effects of the Health Transformation Plan on caesarean section rate in the Islamic Republic of Iran: an interrupted time series. East Mediterr Health J, 25(4), 254-261. doi: 10.26719/emhj.18.044

Romero-Gonzalez, B., Peralta-Ramirez, M. I., Caparros-Gonzalez, R. A., Cambil-Ledesma, A., Hollins Martin, C. J., \& Martin, C. R. (2019). Spanish validation and factor structure of the Birth Satisfaction Scale-Revised (BSS-R). Midwifery, 70, 31-37. doi: 10.1016/j.midw.2018.12.009

Setudezadeh, Fatemeh, \& Yousefinezhadi, Taraneh (2019). The increasing prevalence of cesarean in Iran: How the rate of cesareans could be controlled? Obstetrics \& Gynecology International Journal . Obstet Gynecol Int J., 9(6), 532-535. doi: 10.15406/ogij.2018.09.00402

Shahshahan, Zahra, Heshmati, Bahram , Akbari, Mojtaba , \& Sabet, Fahimeh (2016). Caesarean section in Iran. lancet 388, 29-30.

Skodova, Z., Nepelova, Z., Grendar, M., \& Baskova, M. (2019). Psychometric properties of the Slovak version of the Birth Satisfaction Scale (BSS) and Birth Satisfaction ScaleRevised (BSS-R). Midwifery, 79, 102550. doi: 10.1016/j.midw.2019.102550

Streiner, DL (2003). Starting at the Beginning: An Introduction to Coefficient Alpha and Internal Consistency. J Pers Assess, 80(1), 99-103. doi:

10.1207/S15327752JPA8001_18 
Wijma, K., Wijma, B. , \& Zar, M. . (1998). Psychometric aspects of the W-DEQ: a new questionnaire for the measurement of fear of childbirth. J Psychosom Obstet Gynecol 19(2), 84-97 http://dx.doi.org/10.3109/01674829809048501 PMID:

01674829809638601. doi: http://dx.doi.org/10.3109/01674829809048501

Yousefinezhadi, Taraneh, Mosadeghrad, Ali Mohammad, Arab, Mohammad, Ramezani, Mozhdeh, \& Sari, Ali Akbari. (2017). An Analysis of Hospital Accreditation Policy in Iran. Iranian journal of public health, 46(10), 1347-1358. 
Table 1. Demographic characteristics of participants ( $\mathrm{N}=784)$.

\begin{tabular}{|c|c|c|}
\hline Variable & & $\mathrm{N}(\%)$ \\
\hline \multicolumn{3}{|l|}{ Age } \\
\hline & $<20$ & $51(6.5)$ \\
\hline & $20-29$ & $389(49.6)$ \\
\hline & $30-39$ & $314(40.1)$ \\
\hline & $>40$ & $30(3.8)$ \\
\hline \multicolumn{3}{|l|}{ Education } \\
\hline & Elementary & $127(16.2)$ \\
\hline & High school & $140(17.9)$ \\
\hline & Diploma & $317(40.4)$ \\
\hline & Academic degree & $200(25.5)$ \\
\hline \multicolumn{3}{|l|}{ Job } \\
\hline & Housewife & $704(89.8)$ \\
\hline & Employed & $80(10.2)$ \\
\hline \multicolumn{3}{|c|}{ Monthly household Income } \\
\hline & Less than enough & $273(34.8)$ \\
\hline & Enough or more & $511(65.2)$ \\
\hline \multicolumn{3}{|l|}{ Parity } \\
\hline & Primipara & $319(40.7)$ \\
\hline & Multipara 2 & $270(34.4)$ \\
\hline & Multipara 3 & $195(24.9)$ \\
\hline \multicolumn{3}{|l|}{ Mode of delivery } \\
\hline & Elective Cesarean & $101(12.9)$ \\
\hline & Emergency Cesarean & $146(18.6)$ \\
\hline & Normal Vaginal Delivery & $537(68.5)$ \\
\hline
\end{tabular}


Table 2. Mean, standard deviation and distributional characteristics of individual Iranian BSS-R items. se $=$ standard error of kurtosis $(\mathrm{N}=784)$.

\begin{tabular}{lllllccccc}
\hline & \multicolumn{1}{c}{ Item Content } & Domain* & Mean & SD & Range & Skew & Kurtosis & Se \\
\hline BSS-R 1 & I came through childbirth virtually unharmed & SE & 2.81 & 1.22 & 4 & -0.81 & -0.29 & 0.04 \\
BSS-R 2 & I thought my labour was excessively long & SE & 1.64 & 1.48 & 4 & 0.31 & -1.32 & 0.05 \\
BSS-R 3 & The birthing room staff encouraged me to make decisions & QC & 2.30 & 1.44 & 4 & -0.26 & -1.27 & 0.05 \\
& about how I wanted my birth to progress & & & & & & \\
BSS-R 4 & I felt very anxious during my labour and birth & WA & 1.11 & 1.31 & 4 & 0.85 & -0.53 & 0.05 \\
BSS-R 5 & I felt well supported by staff during my labour and birth & QC & 3.14 & 1.00 & 4 & -1.10 & 0.72 & 0.04 \\
BSS-R 6 & The staff communicated well with me during labour & QC & 3.19 & 0.93 & 4 & -1.06 & 0.64 & 0.03 \\
BSS-R 7 & I found giving birth a distressing experience & SE & 1.79 & 1.42 & 4 & 0.23 & -1.22 & 0.05 \\
BSS-R 8 & I felt out of control during my birth experience & WA & 2.07 & 1.43 & 4 & -0.02 & -1.32 & 0.05 \\
BSS-R 9 & I was distressed during labour & SE & 1.51 & 1.32 & 4 & 0.42 & -0.98 & 0.05 \\
BSS-R 10 & The delivery room was clean and hygienic & QC & 3.37 & 0.82 & 4 & -1.17 & 0.73 & 0.03 \\
\hline
\end{tabular}

*Domain of the Persian BSS-R. SE = Stress experienced during child-bearing, WA = Women's attributes, QC = Quality of Care 
Table 3. Mean, standard deviation, distributional characteristics, and Cronbach's alpha of Iranian BSS-R sub-scales and total score $(\mathrm{N}=784)$.

\begin{tabular}{|c|c|c|c|c|c|c|c|c|c|c|c|}
\hline Subscale & Mean & SD & Min & Max & Skew & Kurtosis & se & Persian alpha & $\mathrm{UK}$ alpha ${ }^{\dagger}$ & $\chi^{2}$ & $p$ \\
\hline Stress & 7.75 & 3.89 & 0 & 16 & 0.23 & -0.59 & 0.14 & 0.68 & 0.71 & 0.50 & 0.48 \\
\hline Attributes & 3.19 & 2.27 & 0 & 8 & 0.40 & -0.76 & 0.08 & 0.53 & 0.64 & 1.97 & 0.16 \\
\hline Quality & 12.00 & 3.12 & 0 & 16 & -0.60 & 0.02 & 0.11 & 0.70 & 0.74 & 1.05 & 0.31 \\
\hline Total Score & 22.94 & 7.04 & 3 & 40 & 0.26 & -0.40 & 0.25 & 0.76 & 0.79 & 1.25 & 0.26 \\
\hline
\end{tabular}


Table 4. Confirmatory factor analysis of the Iranian BSS-R.

\begin{tabular}{lccccc}
\hline Model & $\mathrm{N}$ & $\chi^{2}(\mathrm{df})$ & RMSEA & SRMR & CFI \\
\hline 1. One-factor & 784 & $1141.181(35)$ & 0.201 & 0.152 & 0.549 \\
2. Three-factor & 784 & $168.622(32)$ & 0.074 & 0.074 & 0.944 \\
\hline
\end{tabular}


Table 5. Correlations of the Iranian BSS-R sub-scales and total score and comparison with the original United Kingdom BSS-R validation study (Hollins Martin and Martin, 2014).

\begin{tabular}{lccccc}
\hline Scale combination & Farsi $r$ & UK $r$ & Z & $95 \%$ CI & $p$ \\
\hline Stress-Attributes & 0.62 & 0.57 & 1.02 & $(-0.04-0.15)$ & 0.31 \\
Stress-Quality & 0.28 & 0.26 & 0.29 & $(-0.11-0.16)$ & 0.78 \\
Attributes-Quality & 0.12 & 0.35 & 3.24 & $(0.09-0.36)$ & $\mathbf{0 . 0 0 1}$ \\
Total score-Stress & 0.88 & 0.86 & 1.09 & $(-0.01-0.06)$ & 0.28 \\
Total score-Attributes & 0.72 & 0.80 & 2.52 & $(0.02-0.14)$ & $\mathbf{0 . 0 1}$ \\
Totals score-Quality & 0.64 & 0.63 & 0.22 & $(-0.07-0.10)$ & 0.82 \\
\hline
\end{tabular}


Table 6. Comparison of Iranian BSS-R total and sub-scale scores differentiated by Cesarean section type. Standard deviations are in parentheses, degrees of freedom $=245, \mathrm{CI}=$ confidence interval.

\begin{tabular}{|c|c|c|c|c|c|c|c|c|}
\hline BSS-R Scale & $\begin{array}{c}\text { Elective CS } \\
\qquad(\mathrm{N}=101)\end{array}$ & $\begin{array}{c}\text { Emergency CS } \\
\qquad(\mathrm{N}=146)\end{array}$ & $95 \% \mathrm{CI}$ & $t$ & $p$ & Hedges g & Hedges g 95\% CI & Effect size \\
\hline Stress & $9.02(3.57)$ & $6.56(3.78)$ & $1.52-3.40$ & 5.14 & $<0.001$ & 0.66 & $0.40-0.92$ & Medium \\
\hline Attributes & $3.47(2.23)$ & $2.77(2.11)$ & $0.14-1.24$ & 2.48 & 0.01 & 0.32 & $0.06-0.57$ & Small \\
\hline Quality & $12.20(2.96)$ & $11.10(3.22)$ & $0.30-1.89$ & 2.72 & 0.007 & 0.35 & $0.09-0.61$ & Small \\
\hline Total score & $24.68(6.63)$ & $20.44(6.89)$ & $2.52-5.97$ & 4.83 & $<0.001$ & 0.62 & $0.36-0.88$ & Medium \\
\hline
\end{tabular}




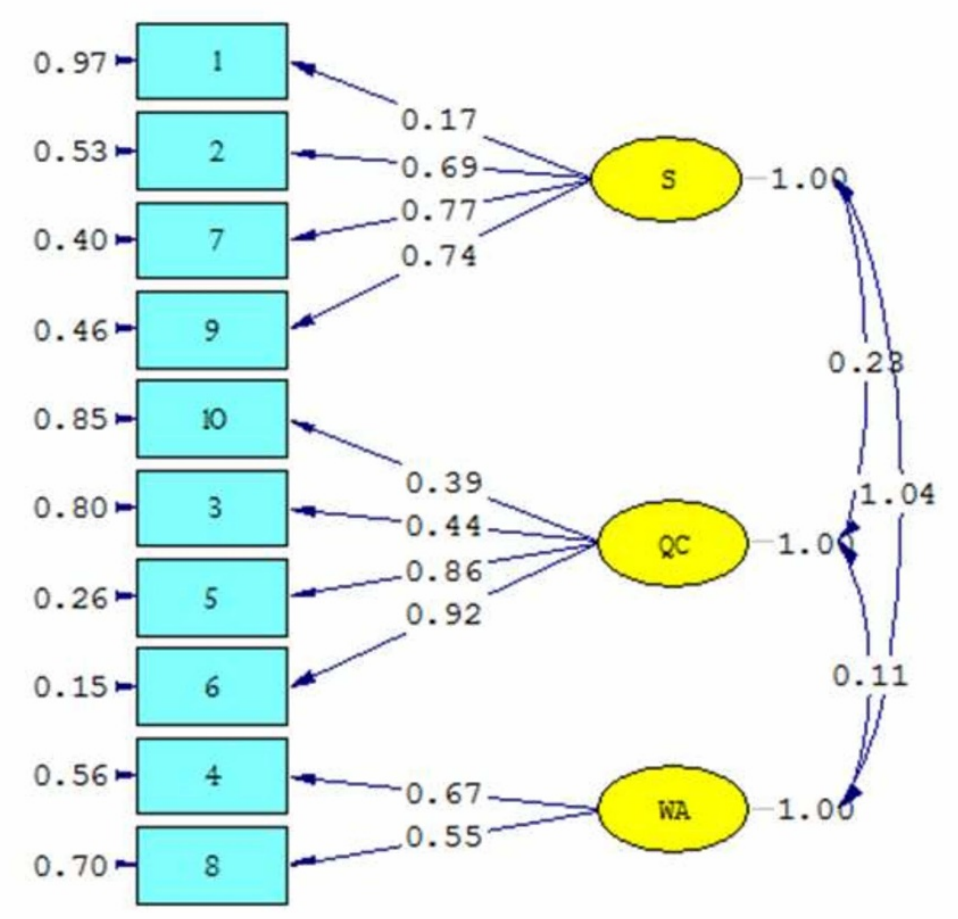

Chi-Square=158.98, df=32, P-value=0.00000, RMSEA $=0.071$ 